\title{
Switching off hysteresis in perovskite solar cells by fine-tuning energy levels of extraction layers
}

\author{
Antonio Guerrero, ${ }^{1 *}$ Agustín Bou, ${ }^{1}$ Gebhard Matt, ${ }^{2}$ Osbel Almora, ${ }^{2}$ Thomas \\ Heumüller, ${ }^{2}$ Germà Garcia-Belmonte, ${ }^{1}$ Juan Bisquert, ${ }^{1}$ Yi Hou,,${ }^{2 *}$ Christoph \\ Brabec $^{2,3 *}$
}

1 Institute of Advanced Materials (INAM), Universitat Jaume I, 12006 Castelló, Spain

2 Institute of Materials for Electronics and Energy Technology (i-MEET), Department of Materials Science and Engineering, Friedrich-Alexander-Universität ErlangenNürnberg (FAU) Martensstr.7, 91058 Erlangen, Germany

3 Bavarian Center for Applied Energy Research (ZAE Bayern), Immerwahrstrasse 2, 91058 Erlangen, Germany

Email: aguerrer@uji.es, yi.hou@ fau.de, christoph.brabec@fau.de

1 March 2019

\begin{abstract}
Lead halide perovskites often suffer from a strong hysteretic behavior on their $j-V$ response in photovoltaic devices that has been correlated with slow ion migration. Electron extraction layer has often been pointed as the main responsible for the observed hysteretic behavior. In this work we focus on three hole transport layers with welldefined HOMO levels and interestingly the hysteretic behavior is markedly different. Here we show that an adequate energy level alignment between HOMO level of the extraction layer and valence band of the perovskite not only reduces degradation of the HTL but also totally suppresses the hysteresis avoiding charge accumulation at the interfaces. Numerical simulation suggests that formation of an injection barrier at the organic/perovskite hetero-interface can be one mechanism causing hysteresis. The suppression of such barriers may require novel design rules for interface materials. Overall, this work highlights that both external contacts need to be carefully optimized in order to obtain hysteresis-free perovskite devices.
\end{abstract}

\section{Keywords}

Perovskite, hysteresis, hole extraction layer, energy barrier, charge accumulation 
Hybrid organic-inorganic perovskite materials have been recently positioned as a strong alternative for high power conversion efficiency (PCE) photovoltaic devices at low costs. ${ }^{[1,2]}$ The perovskite film can be produced by solution process methods that represent a real economic advantage over commercially available technologies. Impressive record efficiencies of $\approx 22 \%$ have been reported using small area devices by spin coating processes. ${ }^{[1,3]}$

A strong hysteretic behavior in the current density-voltage $(j-V)$ response has been observed in several device configurations that has been reduced to some extend by advanced interface engineering. ${ }^{[2]}{ }^{[4]}$ The observed hysteresis is related to the chemical and physical interactions of the perovskite layers with the external interfaces used to extract charges. ${ }^{[5]}$ In the presence of an electrical field created by an external voltage bias or light, ions migrate across the bulk of the perovskite layer reaching the external interfaces where they accumulate. ${ }^{[6]},{ }^{[7]},{ }^{[8]}$ Ion accumulation at these interfaces has been detected by different techniques with spatial resolution in the migration axis such as TOF-SIMS, XRD or PL confocal microscopy. [9],[10] Once ions reach the external contacts they will respond differently to an applied voltage sweep rate used for the $j$ - $V$ measurement depending on the chemical interactions with the external contact leading to distinct hysteretic behavior. ${ }^{[11]}$ In the worst case scenario the interaction with the external contacts leads to irreversible reactions that modify the recombination regime reducing the PCE of the devices. ${ }^{[5,12-14]}$ [14]

Although hysteresis has been described in some degree of detail, the precise role of the external contacts still remains under debate. We have recently shown that the replacement of metal oxides by a monolayer of fullerene derivatives that can selfassemble on the top of the oxide is sufficient to significantly reduce the hysteresis allowing to obtain efficiencies of up to $17.1 \%{ }^{[15]}$ The chemical interactions between the iodide migrating ions and the external contacts is crucial at determining whether ions will continue to be mobile once they reach the external interfaces or will be retained irreversibly. ${ }^{[16]}$ For example, whereas $\mathrm{TiO}_{2}$ leads to weak chemical interactions with iodide ions, oxidized Spiro-OMeTAD can irreversibly react with iodide inducing increases in the series resistance of the device. ${ }^{[5]}$ So far, a simple answer on the effect of the perovskite/HTL interface has not been provided in the literature since the most studied HTL, Spiro-OMeTAD, includes in the formulation additives such as LiTFSi and tert-butyl pyridine. These additives modify the energetics of the interface ${ }^{[17]}$ and also contribute towards the hysteresis of the system by providing extrinsic $\mathrm{Li}^{+}$ions which can migrate through the perovskite layer. ${ }^{[18]}$ Importantly, a literature search on the use of HTL in the absence of additives clearly shows that hysteresis has not been studied in detail as can be noted from data shown as the supporting information (Table SI1).

In the current work we aim to understand whether energy level alignment at the external contacts has any impact on the hysteresis behavior of perovskite solar cells. Three different hole transport layers have been tested each containing a different offset in energy between the HOMO level and the valence band of the perovskite. Adequate 
energy level matching of the perovskite layer with the polymer PDCBT not only reduces the hysteresis but also increases the efficiency to $19.2 \%$ for this series of un-doped HTLs with reduced degradation under illumination conditions.

\section{Results}

Devices have been prepared in the structure ITO/SAM-fullerene/MAPbI $3 / \mathrm{HTL} / \mathrm{Metal}$ (Fig. 1a) following the best configuration recently published by our group. ${ }^{[19]}$ Here we study a system composed by an electron transport layer including a fullerene derivative known to be able to suppress hysteresis. ${ }^{[15]}$ Alternatively, three different HTLs have been tested with chemical structures shown in Fig. 1b., each contain different energetic position of the HOMO levels as reported previously P3HT $~ 5.0 \mathrm{eV}, \mathrm{DPP}$-derivative $5.3 \mathrm{eV}, \mathrm{PDCBT} \sim 5.4 \mathrm{eV} .{ }^{[19]}$ The valence band energy for $\mathrm{MAPbI}_{3}$ prepared in this work is measured to be $\sim 5.4 \mathrm{eV}$, thus making PDCBT the only HTL in the series that provides a sufficient deep HOMO level perfectly matching the one of the perovskite. Therefore, we aim to understand the relationship of the energy level matching of perovskites with external contacts and hysteresis in the $J$ - $V$ response.

Hysteresis usually magnifies when the sweep rate is increased as migrating ions are not able to follow the electrical field. ${ }^{[5]}$ For this reason, most articles reporting low hysteresis minimize it by measuring under very low scan rates (i.e. $10-50 \mathrm{mV} / \mathrm{s}$ ). ${ }^{[1,20]}$ Here we analyze dynamic hysteresis consisting on observed asymmetry of $j$ - $V$ response curve, depending on the direction and rate of the voltage scan. Representative data is shown in Fig. 1 for PDCBT, DPP and P3HT. We note that devices fabricated with PDCBT do not show hysteresis in the range of $10 \mathrm{mV} / \mathrm{s}$ to $1000 \mathrm{mV} / \mathrm{s}$, further data is shown as supporting information. Alternatively, both DPP derivative and P3HT show strong hysteresis when measured at high scan rates. Indeed, the strongest hysteretic behavior is observed for P3HT, the system that nominally shows the largest difference in energy levels of $0.4 \mathrm{eV}$.

If we are to study the effect of the energy offset between the perovskite layer and the HTL we would like to use two case examples one where this difference is negligible (PDCBT) and one with maximized offset (P3HT). However, we noted that devices based on P3HT degrade very fast impeding further electrical measurements with no degradation. Supporting information (Fig. SI2) shows comparative maximum power point tracking under 1 sun illumination of perovskite devices containing two HTLs. Whilst devices containing PDCBT shows a small PCE variation $( \pm 5 \%)$ during the first hour devices with P3HT already reduce the efficiency by $30 \%$. In addition, after $65 \mathrm{~h}$ of continuous illumination devices containing PDCBT are able to recover most of the initial efficiency when the system is let to relax under dark conditions and no applied bias. Alternatively, P3HT degrades irreversibly and relaxation in the dark does not lead to a PCE recovery. We have previously proposed that the position of the HOMO level determines de reactivity towards the redox system $\mathrm{I}^{-} / \mathrm{I}_{2}(\sim 5.1 \mathrm{eV}) .{ }^{[19]} \mathrm{In}$ this work we 
will show that the offset in energy level alignment will not only determine the stability of the interface but also will dictate the hysteresis in the $j-V$ curves response. Due to fast degradation of devices containing P3HT the rest of the electrical characterization is carried out for devices containing PDCBT and DPP derivative as HTLs.

\section{a)}

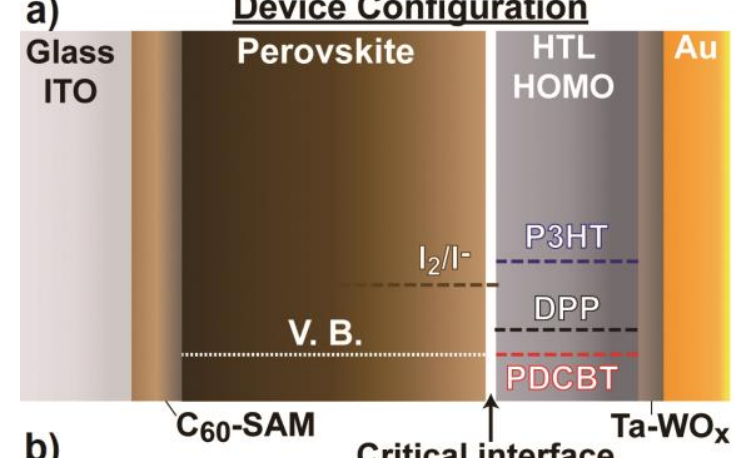

b)

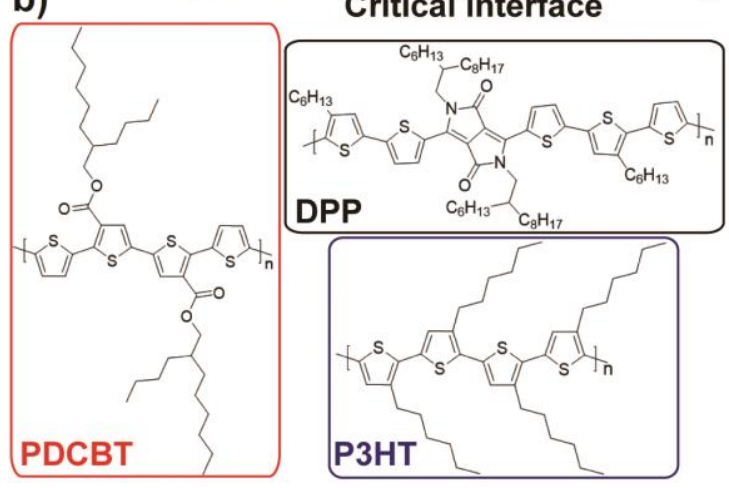

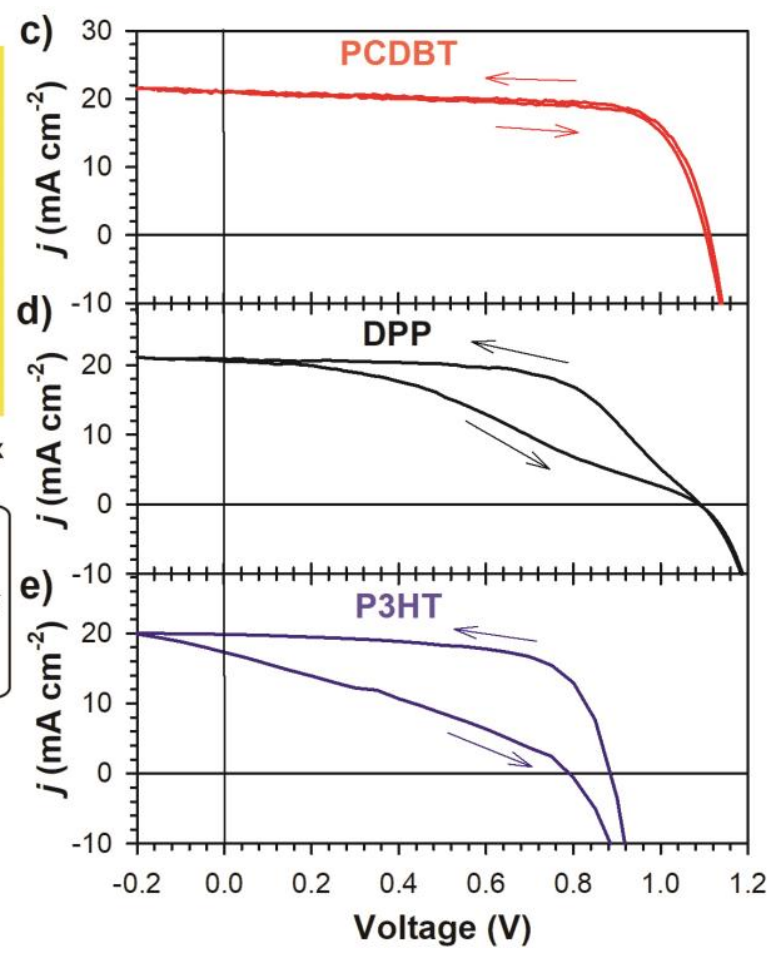

Figure 1: a) Device configuration used in this study where the HTL is the only parameter that has been modified. b) Chemical structure of the different HTL used in this study. Electrical response comparison of devices containing different HTLs: c) PDCBT, d) the DPP derivative and e) P3HT. Devices have been measured at 1 sun light intensity at a relatively fast scan rate of $1 \mathrm{~V} / \mathrm{s}$.

In order to understand the origin of the hysteretic behavior we carry out Impedance Spectroscopy (IS) measurements as a powerful technique for monitoring electrode polarization of migrating ions in perovskite research. ${ }^{[16,21]}$ In this technique, a small AC voltage perturbation is applied in a working device at a given DC bias and the differential current output provides information on resistive and capacitive processes simultaneously. In this respect, electronic and ionic processes are being decoupled as these take place at different time scale (or frequency scale) in perovskite devices. At very low frequencies of $1 \mathrm{~Hz}$ or lower, also electrode polarization has been detected in perovskite solar cells leading to slow capacitive currents in the regime of seconds. Electrode polarization requires the stabilization of the diffusing ion across the interface. Once migrating ions reach the semiconductor/electrode interface the external contact has to compensate the ionic surplus charge to maintain electroneutrality. Depending on the polarity, either electrons or holes have to be provided for compensation. Under 
illumination, the capacitive current adds to the photocurrent leading to hysteretic behavior due to the different transport kinetics of ions and photogenerated carriers. This ion distribution is responsible for an ionic capacitance that has been detected by IS measured in the dark with values in the order of $1-10 \mu \mathrm{F} / \mathrm{cm}^{2}$, typical for Helmholtz layers in liquid electrolytes.

Here were carried out measurements under dark conditions using different DC applied bias with an AC frequency range of $10 \mathrm{mHz}$ to $1 \mathrm{MHz}$. Under these conditions, as shown in Fig. 2a devices fabricated with PDCBT show very low capacitance values of $0.08 \mu \mathrm{F} / \mathrm{cm}^{2}$ at $0.36 \mathrm{~Hz}$ (Fig. 2c) indicating that accumulation of ions is not observed in the range $0 \mathrm{~V}$ and $500 \mathrm{mV}$. Interestingly, ionic capacitance only becomes visible at higher bias voltage $V_{a p p}$ with capacitance values at $0.1 \mathrm{~Hz}$ of $0.7 \mu \mathrm{F} / \mathrm{cm}^{2}$ at $0.8 \mathrm{~V}$ and 17 $\mu \mathrm{F} / \mathrm{cm}^{2}$ at $1 \mathrm{~V}$.

Current-voltage measurements carried out in the dark are a useful technique to detect capacitive currents induced by migrating ions. ${ }^{[5,21]}$ A capacitive current depends linearly on the scan rate following the expression $J_{c a p}=d Q^{l} d t=C d V / d t=C s$ under the assumption of a voltage-independent capacitance. ${ }^{[22]}$ For a device containing PDCBT, relatively fast voltage cycling at $1 \mathrm{~V} / \mathrm{s}$ in the dark does not show significant capacitive current contributions from electrode polarization of migrating ions (Fig. 2d). A linear correlation of $J$ and scan rate is confirmed (Fig. 2e) and the extracted capacitance values of $\mathrm{C} \approx 0.03 \mu \mathrm{F} / \mathrm{cm}^{2}$ at $0.2 \mathrm{~V}$ correlate well with the capacitance extracted from capacitance-frequency spectroscopy (Fig. 2c). Furthermore, the device does not present significant hysteresis during the measurement of the $j-V$ curve under illumination conditions.

Alternatively, devices fabricated with the DPP derivative show a typical ionic capacitance in the range between $0-1.0 \mathrm{~V}$ (Fig. $2 \mathrm{~b}$ ) with calculated values from the linear relationship of $j$ vs scan rate of $\mathrm{C} \approx 3.4 \mu \mathrm{F} / \mathrm{cm}^{2}$ at $0.2 \mathrm{~V}$. Therefore, in this second situation, a remarkable capacitive current density is observed under dark conditions which almost negligibly increases with applied bias in the low frequency region as observed in the Capacitance-Voltage measurements. The presence of such large capacitance values typical arises from electrode polarization. We have highlighted previously that this behavior is related to the hysteresis phenomena under illumination conditions. ${ }^{[16]}$ 

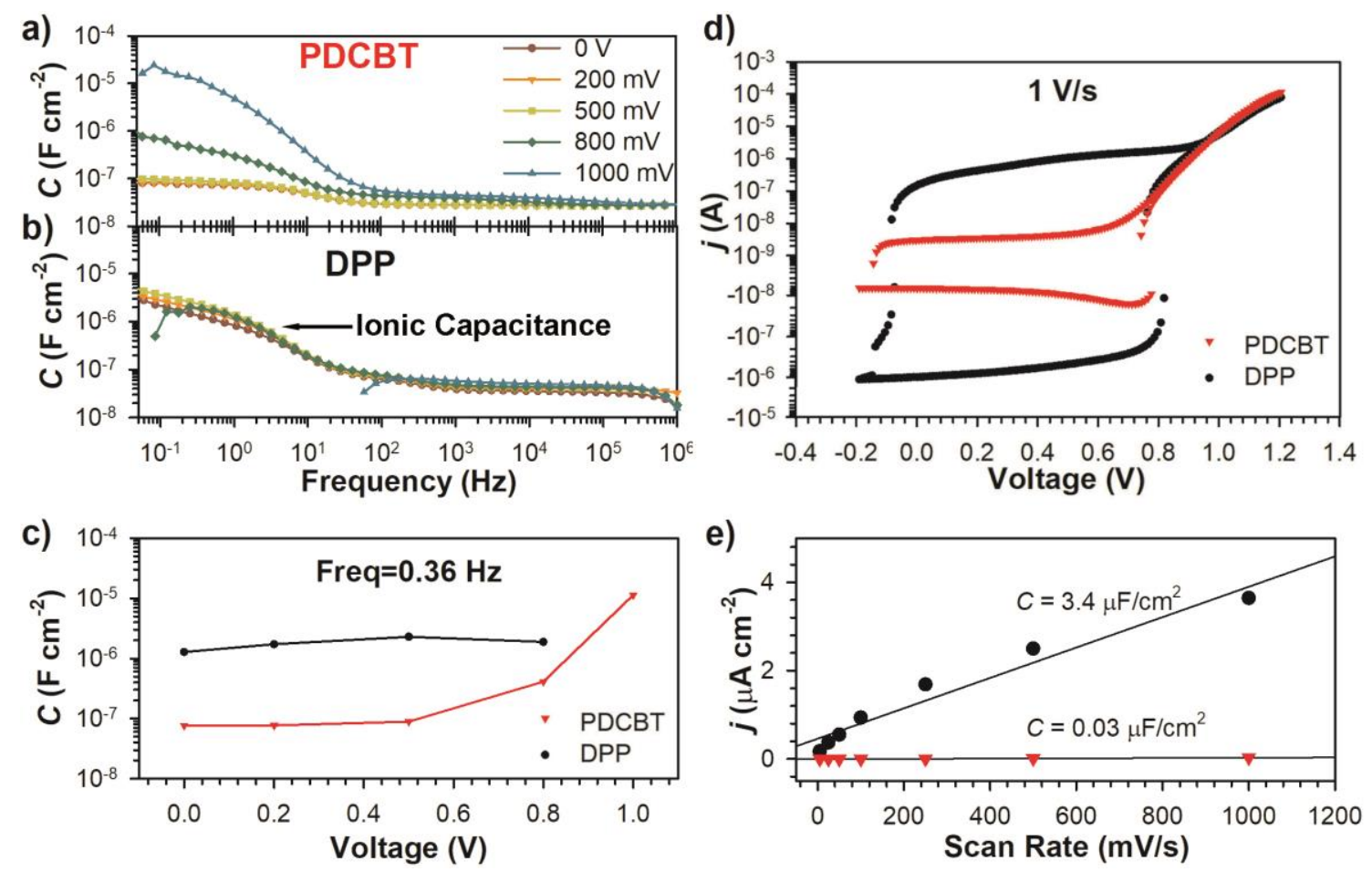

Figure 2: Capacitance vs frequency plot of devices measured under dark conditions as a function of the DC applied bias for devices containing PDCBT a) and DPP b). c) Extracted $C$ - $V$ values in the low frequency region $(0.36 \mathrm{~Hz})$. d) Current-voltage curves measured in the dark at a scan rate of $1 \mathrm{~V} / \mathrm{s}$ highlighting the differences in capacitive currents. e) Calculated capacitance from linear region of current versus scan rate for $j-V$ measurements in the dark at $0 \mathrm{~V}$.

Our results show that the modification of the HTL interface provokes a major modification of the hysteresis and capacitance response of the perovskite solar cell. This behavior indicates that the new self-assembled fullerene derivative layer on the top of the oxide remarkably suppresses polarization and hysteresis. Indeed most previous studies showed the dominant electrical polarization and ion accumulation occurring at the ETL oxide surface. We now outline a detailed physical model to describe the major variations observed at the contact between the perovskite and the HTL based on the variation of energetic and kinetic properties according to the difference of HTL workfunction.

The perovskite/PDCBT interface with perfectly aligned energy levels between the perovskite valence band (VB) and the HOMO level of the HTL is compared in Fig. 3a under equilibrium conditions in the dark. A second situation is shown in Fig. 3b with an offset of $0.3 \mathrm{eV}$ between the HOMO of the HTL and the perovskite VB, to resemble the situation observed for the DPP derivative. Thus, the built-in potential $\left(V_{b i}\right)$ of the aligned device (PDCBT) is $0.3 \mathrm{~V}$ higher than the not-aligned one (DPP). This difference in $V_{b i}$ is confirmed qualitatively in devices by Mott-Schottky analysis of the capacitance in the high frequency region $(10 \mathrm{kHz})$ as shown in the supporting information. In addition, 
qualitatively the diagram is supported by kelvin probe measurements on devices containing $\mathrm{TiO}_{2}$ and Spiro-OMeTAD. ${ }^{[23]}$ Here, the energy diagram is built by numerical solution of Poisson equation, as well as drift-diffusion equations for free carriers and mobile ions, and the variation of HOMO level has been amplified (with respect to experimental value $0.13 \mathrm{eV}$ ) for clarity of discussion. Detailed parameters information can be found as supporting information. The discontinuity of the perovskite/DPP at any applied voltage is just the result of the difference between the HOMO of the HTL and VB of the perovskite, which remains constant as the vacuum level is continuous. In general, the field drops very sharply within the first $10 \mathrm{~nm}$ at the organic layer due to the small dielectric constant $(\varepsilon \sim 3)$ in comparison to that of perovskite $(\varepsilon \sim 26){ }^{[24]}$ Alternatively, the depletion region extends further inside the perovskite layer for both systems. When an external voltage bias $V_{a p p}=1.1 \mathrm{~V}$ is applied to the device (Fig. $3 \mathrm{c}$ and $3 \mathrm{~d}$ ), the Fermi level of electrons raises towards the conduction band (CB) and causes accumulation regime when the $V_{a p p}$ exceeds the $V_{b i}$, creating a large concentration of electrons at the surface that is accompanied by ionic charge accumulation, as well as an accumulation of holes in the HTL. Note that the applied electrical field induces an inversion on the bending and now the transport of negative ions towards the perovskite/HTL interface is favoured. Due to the difference in energy levels between PDCBT and DPP, the accumulation for devices containing PDCBT occurs at higher voltage. Therefore it is clear that the charge at the perovskite-DPP interface is always higher than the charge at the perovskite-PDCBT, leading to higher accumulation, 20-40 times higher according to the simulation with only $0.3 \mathrm{~V}$ offset.

Very importantly, for both HTLs electrons and ions diffuse, however, in the case of DPP the ion and electron concentration close to the Perovskite/HTL interface is much higher. Due to the presence of a kink and a subsequent energy barrier at this heterointerface between perovskite and the DPP derivative, holes injection from DPP into perovskites is suppressed, leading to a narrow and highly charged space charge accumulation layer at the HTL side. As such, a capacitance "across" the interface is formed, which becomes much larger than for PDCBT where electronic charges (holes) can migrate into the perovskite and then stabilize within a few $\mathrm{nm}$ the high amount of iodine ions and electrons that are in the perovskite. In summary capacitive effects observed above are explained by this model, and also the occurrence of larger hysteresis in DPP, due to the increased amount of ion motion under transient conditions 

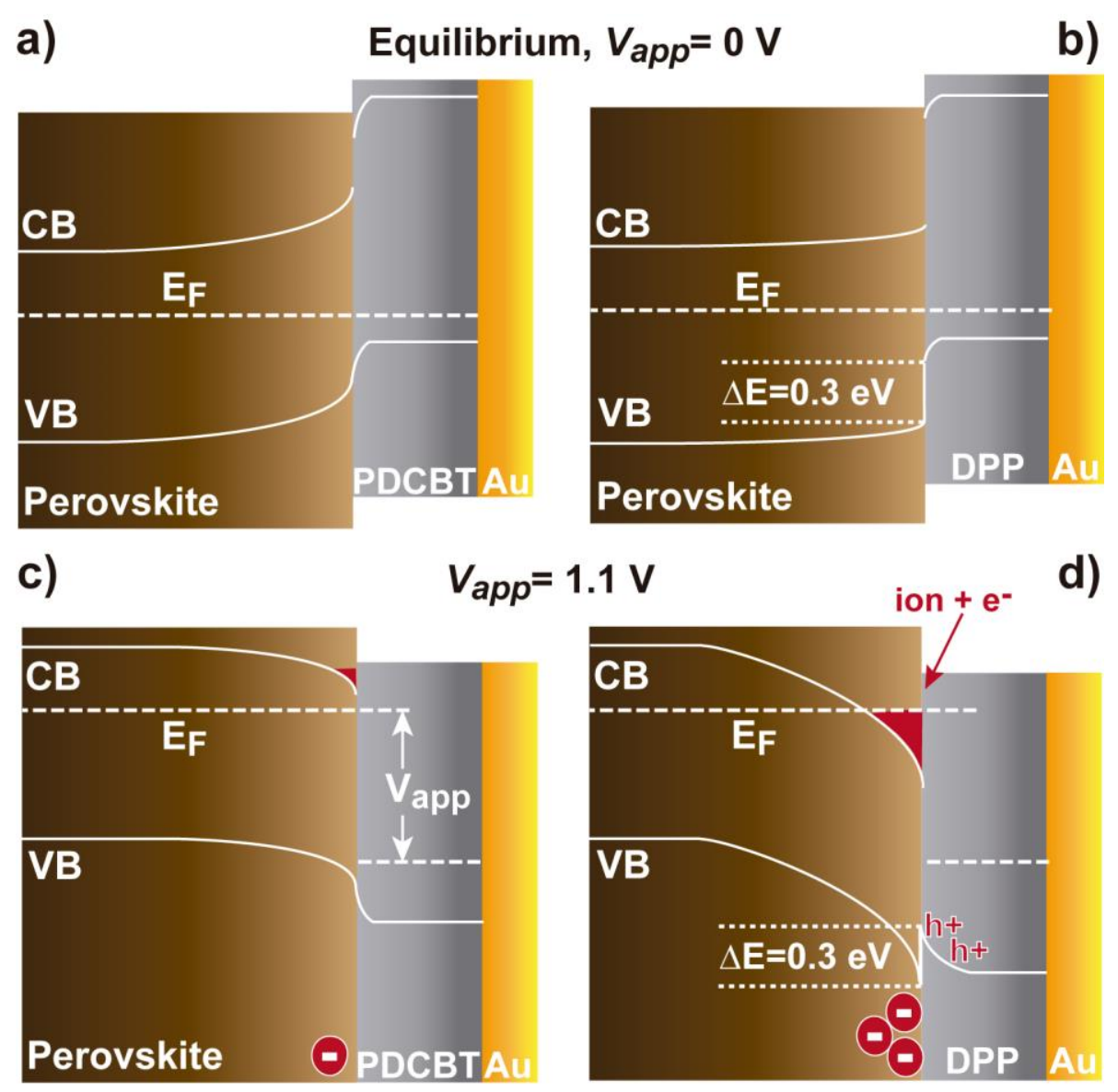

Figure 3: Band diagram scheme of the simulated perovskite/HTL interface in the dark. a) and b) At equilibrium conditions $V_{a p p}=0 \mathrm{~V}$ and c) and d) under applied voltage bias of $1.1 \mathrm{~V}$. a) and c) Represents a perovskite/PDCBT interface with energy level alignment of valence band of perovskite with HOMO level of HTL when no hysteresis is observed. b) and d) A perovskite/DPP interface represents a system with and energy level offset of $0.3 \mathrm{~V}$ that shows strong hysteretic behavior.

It is significant to note that at $V_{a p p}=0 \mathrm{~V}$ none of the systems are in accumulation regime and the fact that a large capacitance is observed for the device containing DPP might be related with a pre-treatment leading to strong chemical interactions of migrating ions with the HTL. In particular, we have previously reported that once devices are illuminated or a voltage bias has been applied the migrating ions can irreversibly interact with the contacts. ${ }^{[5,12,14]}$ In this case, the PDCBT and DPP derivatives are chemically very different and iodide ions can interact chemically different in both cases leading to different kinetics of ion migration. To further support this we note that as the applied bias is increased and as the system goes into accumulation regime, a negative capacitance in the low frequency region is observed, spectra are shown as supporting information. This negative capacitance, which shows an inductive loop in the Nyquist plots, has been related to the charging of ions and carriers at the interfaces. ${ }^{[25]}$ Therefore, the slow kinetics of the ions lead to a slow discharging of 
the capacitance at the interface, creating capacitive currents that produce hysteresis.

Overall the simulation results help to understand the $j$ - $V$ curves hysteretic behavior and the ionic capacitance observed in the impedance results. In devices fabricated with an HTL that provides adequate energy levels alignment charging of the interface tends to be minimized. Alternatively, devices with an offset in energy level alignment between HTL and perovskite mobile ions will be closer to the external interfaces leading to large ionic capacitance values and large hysteresis during characterization by $j-V$ analysis.

\section{Conclusions}

The present work highlights the importance of energy level alignment between the perovskite and the external contacts. Devices with HOMO levels of the HTL with lower values in energy than the VB of the perovskite show high ionic contribution to the electrical response. We highlight the presence of a charge injection barrier at the organic-perovskite interface as one major origin for hysteresis. Such single-sided or double sided barriers can be prevented by carefully designing novel interface materials. We conclude that, in the presence of diffusing ions, a perfect energy level alignment is required for hysteresis-free electrical response. In addition, this energy level mismatch could also be related with accelerated degradation and will be the subject of further studies. i.e. via migrating ions with a strong driving force diffuse into the HTL doping the material.

\section{Experimental Section}

Materials and precursor solutions: Unless stated otherwise, all materials were purchased from Sigma Aldrich and used as received. MAI and $\mathrm{PbI}_{2}$, was purchased from Lumtec. To form a $1.4 \mathrm{M} \mathrm{MAPbI}_{3}$ precursor solution, $\mathrm{PbI}_{2}$ and $\mathrm{MAI}$ were mixed with a mole ratio of 1:1 in a mixture of DMF and DMSO (4:1 v/v). The DPP and PDCBT were separately dissolved in chloroform and chlorobenzene at concentrations of $5 \mathrm{mg} / \mathrm{mL}$, and followed by stirring on a $70{ }^{\circ} \mathrm{C}$ hot plate for 2 hours. The $2.5 \mathrm{wt} \% \mathrm{Ta}-\mathrm{WO}_{x}$ ink was provided from Avantama Ltd.

Photovoltaic Device fabrication: First, the patterned ITO substrates were ultrasonically cleaned using acetone and isopropanol for 10 minutes each. On each cleaned ITO substrate, a dense and smooth layer of $\mathrm{C}_{60}$-SAM was deposited by dipping the ITO substrate into the $\mathrm{C}_{60}-\mathrm{SAM}$ solution for 24 hours. The as-prepared perovskite precursor solution was filtered using a $0.45 \mu \mathrm{m}$ PTFE syringe filter and coated onto substrates at a speed of 4000 r.p.m for $20 \mathrm{~s}$. The substrate (around $2.5 \mathrm{~cm} \times 2.5 \mathrm{~cm}$ ) was quenched by blowing $\mathrm{N}_{2}$ gas and annealed on a hot plate at $110 \circ \mathrm{C}$ for $10 \mathrm{~min}$. The prepared polymer HTMs were spin-coated at 1000 r.p.m for $35 \mathrm{~s}$ and annealed at $70{ }^{\circ} \mathrm{C}$ for 5 min. Subsequently, Ta-WO ${ }_{x}$ was coated onto polymer HTMs at a speed of 2000 r.p.m for 30 s. Finally, a 100-nm-thick Au counter electrode was deposited onto a holetransporting layer through a shadow mask by thermal evaporation. 
Film and Device characterization: $j-V$ characteristics of all the devices were measured using a source measurement unit from BoTest. Illumination was provided by a Newport Sol1A solar simulator with AM 1.5G spectrum and light intensity of 100 $\mathrm{mWcm}^{-2}$, which was determined by a calibrated crystalline Si-cell. During device characterization, a shadow mask with an opening of $10 \mathrm{~mm}^{2}$ was used. For $j$ - $V$ measured at different scan rates and Impedance spectroscopy measurements were performed using an Autolab PGSTAT-30 equipped with a frequency analyzer module. IS was carried out under dark conditions at different DC bias from $0 \mathrm{~V}$ up to $1.5 \mathrm{~V}$.

\section{Acknowledgements}

We thank financial support by MINECO of Spain ${ }^{[24]}$ under project (MAT201676892-C3-1-R), and Generalitat Valenciana (ACOMP/2015/105). A. G. would like to thank the Spanish Ministerio de Economía y Competitividad for a Ramón y Cajal Fellowship (RYC-2014-16809). Financial support was provided by the Soltech Initative, the HI-ErN projects funded by the Bavarian Ministry for Economy and the Excellence Cluster "Engineering Advanced Materials " from the DFG.

\section{References}

[1] M. Saliba, T. Matsui, K. Domanski, J.-Y. Seo, A. Ummadisingu, S. M. Zakeeruddin, J.-P. Correa-Baena, W. R. Tress, A. Abate, A. Hagfeldt, M. Grätzel, Science 2016, 354, 206.

[2] H. Tan, A. Jain, O. Voznyy, X. Lan, F. P. García de Arquer, J. Z. Fan, R. QuinteroBermudez, M. Yuan, B. Zhang, Y. Zhao, F. Fan, P. Li, L. N. Quan, Y. Zhao, Z.-H. Lu, Z. Yang, S. Hoogland, E. H. Sargent, Science 2017, 355, 722.

[3] N. E. Chart 2018, https://www.nrel.gov/pv/assets/images/efficiency-chart.png

[4] Y. Hou, W. Chen, D. Baran, T. Stubhan, N. A. Luechinger, B. Hartmeier, M. Richter, J. Min, S. Chen, C. O. R. Quiroz, N. Li, H. Zhang, T. Heumueller, G. J. Matt, A. Osvet, K. Forberich, Z.-G. Zhang, Y. Li, B. Winter, P. Schweizer, E. Spiecker, C. J. Brabec, Adv. Mater. 2016, 28, 5112.

[5] J. Carrillo, A. Guerrero, S. Rahimnejad, O. Almora, I. Zarazua, E. Mas-Marza, J. Bisquert, G. Garcia-Belmonte, Adv. Energy Mater. 2016, 6, 1502246.

[6] O. Almora, A. Guerrero, G. Garcia-Belmonte, Appl. Phys. Lett. 2016, 108, 043903.

[7] W. Tress, N. Marinova, T. Moehl, S. M. Zakeeruddin, M. K. Nazeeruddin, M. Gratzel, Energy Environ. Sci. 2015, 8, 995.

[8] R. Gottesman, P. Lopez-Varo, L. Gouda, Juan A. Jimenez-Tejada, J. Hu, S. Tirosh, A. Zaban, J. Bisquert, Chem 2016, 1, 776.

[9] T. Zhang, X. Meng, Y. Bai, S. Xiao, C. Hu, Y. Yang, H. Chen, S. Yang, J. Mat. Chem. A 2017, 5, 1103; C. Li, S. Tscheuschner, F. Paulus, P. E. Hopkinson, J. 
Kießling, A. Köhler, Y. Vaynzof, S. Huettner, Adv. Mater. 2016, 28, 2446.

[10]C. Li, A. Guerrero, Y. Zhong, A. Gräser, C. A. M. Luna, J. Köhler, J. Bisquert, R. Hildner, S. Huettner, Small 2017, 1701711.

[11]S. Ravishankar, O. Almora, C. Echeverría-Arrondo, E. Ghahremanirad, C. Aranda, A. Guerrero, F. Fabregat-Santiago, A. Zaban, G. Garcia-Belmonte, J. Bisquert, J. Phys. Chem. Lett. 2017, 8, 915.

[12] J.-P. Correa-Baena, S.-H. Turren-Cruz, W. Tress, A. Hagfeldt, C. Aranda, L. Shooshtari, J. Bisquert, A. Guerrero, ACS Energy Lett. 2017, 2, 681.

[13]K. Domanski, B. Roose, T. Matsui, M. Saliba, S.-H. Turren-Cruz, J.-P. CorreaBaena, C. Roldan Carmona, G. Richardson, J. Foster, F. De Angelis, J. Ball, a. petrozza, N. Mine, M. K. Nazeeruddin, W. Tress, M. Gratzel, U. Steiner, A. Hagfeldt, A. Abate, Energy Environ. Sci. 2017, 10, 604.

[14]A. Guerrero, J. You, C. Aranda, Y. S. Kang, G. Garcia-Belmonte, H. Zhou, J. Bisquert, Y. Yang, ACS Nano 2016, 10, 218.

[15] Y. Hou, S. Scheiner, X. Tang, N. Gasparini, M. Richter, N. Li, P. Schweizer, S. Chen, H. Chen, C. O. R. Quiroz, X. Du, G. J. Matt, A. Osvet, E. Spiecker, R. H. Fink, A. Hirsch, M. Halik, C. J. Brabec, Adv. Mat. Interfaces 2017, 4, 1700007.

[16]H.-S. Kim, I.-H. Jang, N. Ahn, M. Choi, A. Guerrero, J. Bisquert, N.-G. Park, J. Phys. Chem. Lett. 2015, 6, 4633.

[17] G. Boschloo, L. Häggman, A. Hagfeldt, J. Phys. Chem. B 2006, 110, 13144.

[18]Z. Li, C. Xiao, Y. Yang, S. P. Harvey, D. H. Kim, J. A. Christians, M. Yang, P. Schulz, S. U. Nanayakkara, C.-S. Jiang, J. M. Luther, J. J. Berry, M. C. Beard, M. M. Al-Jassim, K. Zhu, Energy Environ. Sci. 2017, 10, 1234.

[19] Y. Hou, X. Du, S. Scheiner, D. P. McMeekin, Z. Wang, N. Li, M. S. Killian, H. Chen, M. Richter, I. Levchuk, N. Schrenker, E. Spiecker, T. Stubhan, N. A. Luechinger, A. Hirsch, P. Schmuki, H.-P. Steinrück, R. H. Fink, M. Halik, H. J. Snaith, C. J. Brabec, Science 2017, DOI: 10.1126/science.aao5561.

[20] K. T. Cho, S. Paek, G. Grancini, C. Roldan-Carmona, P. Gao, Y. Lee, M. K. Nazeeruddin, Energy Environ. Sci. 2017, 10, 621.

[21]O. Almora, I. Zarazua, E. Mas-Marza, I. Mora-Sero, J. Bisquert, G. GarciaBelmonte, J. Phys. Chem. Lett. 2015, 6, 1645.

[22]P. Lopez-Varo, J. A. Jiménez-Tejada, M. García-Rosell, S. Ravishankar, G. GarciaBelmonte, J. Bisquert, O. Almora, Adv. Energy Mater. 2018, 1702772.

[23] V. W. Bergmann, Y. Guo, H. Tanaka, I. M. Hermes, D. Li, A. Klasen, S. A. Bretschneider, E. Nakamura, R. Berger, S. A. L. Weber, ACS Appl. Mater. Interfaces 2016, 8, 19402.

[24] O. Almora, L. Vaillant-Roca, G. Garcia-Belmonte, Revista Cubana de Física 2017, 
$34,58$.

[25]E. Ghahremanirad, A. Bou, S. Olyaee, J. Bisquert, J. Phys. Chem. Lett. 2017, 8, 1402. 